\title{
Coagulation Activities of Patients on Psychotropic Drugs in Nigeria
}

\author{
Afolabi O.I ${ }^{1}$, Adedire O.A ${ }^{2}$, Oke O.T ${ }^{2}$, Omisakin C.T ${ }^{3, *}$, Esan A.J ${ }^{3}$ \\ ${ }^{1}$ Deparment of Medical Laboratory Services, General Hospital, Oke-Imesi Ekiti, Nigeria \\ ${ }^{2}$ Department of Haematology and Blood Transfusion, Obafemi Awolowo University Teaching Hospital, Ile - Ife Nigeria \\ ${ }^{3}$ Department of Haematology and Blood Transfusion, Federal Medical Centre, Ido - Ekiti, Nigeria \\ *Corresponding author: christophertop@yahoomail.com
}

Received March 10, 2014; Revised March 25, 2014; Accepted March 30, 2014

\begin{abstract}
The study is being aimed to assess Prothrombin Time and Activated Partial Thromboplastin Time activity in patients on psychotropic drugs. Fifty (50) samples were used, Forty (40) are from the patients on psychotropic drugs while ten (10) are apparently healthy individual that serves as control. The results obtained showed thatPT and APTT of the test subject have a mean value of $26.01 \pm 11.04$ in compared with the control subject showed a mean value $11.80 \pm 1$.3. While the result obtained from APTT test subject showed a mean value of $36.70 \pm 17.00$ compared with the control subject that have a mean value of 36.7.0 \pm 3.81 . When these results were compared statistically significant difference were observed $\mathrm{P}<0.01$ which indicate a state of prolongation in $\mathrm{PT}$ and APTT.
\end{abstract}

\section{Keywords: prothrombin psychotropic drugs, thromboplastin}

Cite This Article: Afolabi O.I, Adedire O.A, Oke O.T, Omisakin C.T, and Esan A.J, “Coagulation Activities of Patients on Psychotropic Drugs in Nigeria.” American Journal of Medical Sciences and Medicine, vol. 2, no. 2 (2014): 41-43. doi: 10.12691/ajmsm-2-2-3.

\section{Introduction}

Blood coagulation can be traced back to about 400BC and the fathers medicine; Hippocrates, Plato, Aristole, Celsus and Galen. Hippocrates was the first person recognized blood of a wounded soldier congeale as if cooled, as well as bleeding from wound stopped, as skin covered the blood. If the skin removed bleeding stated again but Aristole noted that blood cooled when removed from the body when initiated decay resulting in the congealing of the blood, if fibres were removed, there was no clothing [23]. It was not believed to have any physiologic or pathology significant, until a surgeon, recognized in 1930s that clothing was haemostatically important in amputation [23]. Therefore, coagulation is a complex process by which blood forms clots. It is an important part of hemostasis, wherein a damaged blood vessel wall is covered by a platelet and fibrin containing clot to stop bleeding and begin repair of the damage vessel [21]. Also Blood coagulation involves a biological amplification system in which relatively few initiation substance sequentially activate the proteolysis of thrombin, thus in turn, converts soluble plasma fibrinogen into fibrin [10]..Coagulation begins almost instantly after an injury to the blood vessel has damages the endothelium. Exposure of the blood to protein such has tissue factors initiate changes to blood platelet and the plasma protein fibrinogen a clotting factor. Platelets immediately form a plug at the side of injury; this is called primary haemostasis. Secondary haemostasis occurs simultaneously; protein in the blood plasma called coagulation factors or clotting factors, respond in a complex cascade to form fibrin strands, which strengthen plug [6].

Disorder of coagulation can lead to an increase in risk of bleeding hemorrhage or thrombosis. Coagulation disorders are common intensive care patient and may range from isolated thrombocytopenia or prolonged global clotting test to complex defects, such as disseminated intravascular coagulation.

A psychotropic drug is a chemical substance that crosses the blood brain barrier and such acts primarily upon the central nervous system, where it affects brain function, resulting in change in perception, mood, consciousness, cognition and behavior [27].

These substances may be used recreationally to alters one's consciousness as entheogens and Shamanic purpose of these as a tool for studying the therapeutically as medication. Because psychoactive substance bring about subjective change in consciousness and mood that the user may find advantage. Therefore, the use of psychotropic drugs can extremely help reducing psychotic symptoms and agitation in a patient with several mental illnesses. These drugs may produce serious side effect that can range from mild to severe, also they can added as a significant burden, reducing patient quality of life (24). Numbers of harmful and other side effects have been observed, including lower life expectancy, weight gain, enlarged breast, hyperprolactinaemia, Agranulocytosis, Diabetes and inability to sit still [4]. 
Psychotropic drugs use is a practice that dates to Prehistoric time there is archeological evidence of the use of psychotropic drugs mostly likely planks dating back at least 10,000 years, and historical evidence of a culture use over.

Psychotropic drugs have different used and are broken into four major groups [19]

(1) Hallucinogen Drugs (2) Antipsychotic Drugs (3) Depressant Drugs (4) Stimulant Drug.

\subsection{Specific Objective}

The aim of this research work is to assess coagulation activities in patients on psychotropic drugs using PT and APTT with a view to determine susceptibility to hemorrhage or thrombosis.

\section{Materials and Method}

A total number of fifty blood samples were collected, Forty were from patients on psychotropic drugs while the remaining ten were from subjects not on psychotropic drugs to serves as control,The collection of samples were carried out at psychiatric department, Obafemi Awolowo University Teaching Hospital, Ile Ife, Osun State Nigeria. The collected samples were processed at Department of Haematology and Blood Transfusion Obafemi Awolowo University Teaching Hospital Complex Ile -Ife, Osun State. The result obtained after processing were tabulated. Also the mean and standard deviations of the samples was also process using T-Value.

\subsection{Methodology}

\subsubsection{Prothrombin Time (PT) Test}

Plasma is added to a thromboplastin and calcium chloride reagent at $37^{\circ} \mathrm{C}$ and the time taken for a clot to form is measured. The clotting time in seconds is converted to the International Normalized Ratio (INR), usually by reference to a table provided by the manufacturer of reagent [21].

\subsubsection{Activated Partial Thromboplastin Time (APTT) Test}

The patient's plasma provides all the factors for clotting except calcium (removed by citrate) and platelets (removed by centrifugation).Calcium and platelet factor reagent (or a partial thromboplastin) is added to the patient's plasma, and the time of clot formation is noted [21].

\section{Results}

In this study, fifty (50) subjects were used, in which forty (40) were test subject while ten (10) were selected as control. Table 1 shows the overall result of PT and APTT + standard deviation. The statistical significant $(\mathrm{P}<0.01)$ results were observed in PT and APTT in patient on psychotropic drugs when compared with the control (26.01 \pm 11.04), (11.80 \pm 1.30$)$, for PT, while (76.83 \pm 17.00), (36.70 \pm 3.81$)$ for APTT.
Table 1. The overall Result of PT and APTT

\begin{tabular}{ccc}
\hline & PT & APTT \\
\hline TEST N-40 & $26.01 \pm 11.04$ & $76.83 \pm 17.00$ \\
CONTROL N-10 & $11.80 \pm 1.30$ & $36.70 \pm 3.81$ \\
P-Value & $\mathrm{P}<0.01$ & $\mathrm{P}<0.01$ \\
\hline
\end{tabular}

\section{Discussion}

Psychotropic drugs are tranquilizer primarily used to manage psychosis [24]. The medication tends to block D2 receptor in the dopamine of the brain. This means that dopamine released in these pathways is less effective ${ }^{(18) .}$

Coagulation is a complex series of enzymatic reaction involving the proteolytic activation of circulatory coagulation factor (Zymogen) and activity of co-factor leading to the production of thrombin which converts soluble plasma fibrinogen into fibrin [10].

According to Conosoet al 1977, coagulation disorder has been proved to arise from the consumption of psychotropic drugs. This can be association with the thrombocytopenia, platelet dysfunction and hepatomegaly.

The result of this study shown prolonged activated Partial Thromboplastin (APTT) in patient sample on psychotropic drugs in relation to the control and the difference seen were statically significant $(\mathrm{P}<0.01)$. This work is in line with the previous work done by Mohammed et al 2005. In their work prolong result was observed in APTT in patient on long time usage of psychotropic drug. It was deducted from their work that the prolongation result observed might be due to the drug induced hemostasis changes. The hemostasis change at this agent is usually associates with immune thrombocytopenia, liver function impairment and coagulation change [3]. The prothrombin time (PT) test of this study show prolonged and differences were seen statistically significant $(\mathrm{P}<0.01)$. This result was in agree with previous work done by Osinobowaleet al 2010. It was deduced from their work that mild pronlonged PT result were observed might due to Pharmacological constitute of some psychotropic drugs especially second generation such as chloropromazine contain anticoagulant.

Also, In this study $28 \%$ shown normal result while $72 \%$ shown abnormal result in PT whereas, 8\% show normal result while 92\% show abnormal result in APTT. Prolonged result observed in PT showed that there is deficiency in extrinsic factors such as (II, V, VII, and X). Also prolonged result observed in APTT showed that there is deficiency in intrinsic factors such as II, IX, X, XI and XII. This may due to the early stage of the use of the drug, differences in prescribing drugs medication and nutritional factors.

In conclusion, it is pertinent for psychiatrist to put into the consideration the effect of psychotropic drugs on hemostasis especially coagulation before and during administration of psychotropic drugs.

\section{References}

[1] Baker, F.J., Silverton R.E. and Pallister C.J. (2000), Introduction to Medical Laboratory Technology. $7^{\text {th }}$ Edition, Bounty, Page 384392. 
[2] Boyd and Alan, (1995), British Medical Journal Psychiatry, Bromocriptie and Psychosis (66) Page 89-95.

[3] Conoso R.T., Hulton R.A. and Deylcin D.A (1977), Chloropromazine Induced Inhibitor of Blood Coagulation American Journal, Haematology 2: 183-191

[4] Dilsaver, S.C. and Alessi, N.T (2000), AntiPsychotic Withdrawal Symptoms, Phenomenology and Pathophysiology. Acts PsychiatryScand, 77(3) Page 241-246.

[5] Emdex, (2010), The Complete Drug Formulary, Healthy Professionals, Lph, 2010/11 Editions, Page 13-31.

[6] Furie, B.C. (2005), Thrombus Formulation Invivo. Journal at Clinical Investigation. 115(12): 355-3365.

[7] Gelder and Michael (2005), "Psychiatry". Oxford University Press Inc. New York. ISBN.

[8] Haggs .S., Spigset .O. and T. G. Soerstrom T.G. (2000), Associated between Venous Thromboembolism and Clozapine. A study of the WHO Database of Adverse Drugs Reaction (355); Page 1115-1156.

[9] Harper and Douglas (2001), "Hallucinate”. Online Etymology Dictionary www. Etvrnoline.com

[10] Hoffbrand, A.V., Pettit J.E and Moses P.A.N (2004), Essential Haematology, $4^{\text {th }}$ Edition, Blackwell, Page 236-273.

[11] Hougie .C. and Cecil .B. (1963), Fundamental of Blood Coagulation in Clinical Medicine, McGraw-Hill, New York 1963.

[12] International Committee for the Nomenclature of Blood Clotting factors (1962), Nomenclature of Blood Clotting factor. British Medical Journal (465) page 17-20.

[13] Leweke F.M., Koethe D. Pahliseh F., Schrieber, D., Gerth C.W., Nolden B.M., Klosterkotter J., and Helimich (2009), Antipsychotic effect of Cnnabidol, European Psychiatry 24 (207) Page 70440-70447.

[14] Macfalene R.G. (1964), An EmzymeCasada In Blood clotting Mechanism and its function as Biological Amplifer nature 202: 498-499.
[15] McKeith; Lan G. (2002), "Dementia with lewyBodie" British Journal of Psychiatry (180) Page 144-147.

[16] Mohammed H.Z. Stanley Zucker, Frederick M., Gails S.R., James A.H., and Andre O.V. (2005), Coagulation Disorder in Chloromazie Treated Patient, Northorpot, New York page 15-16 (Pubmed).

[17] Morawitz P. (1905), "Die ChemiederBlutgerinnung” Ergehn Physiology (4) Page 307-422

[18] Murpy: B.P, Chung Y.C., Park T.W. and McGorry P.D. (2006), Pharmacological Treatment of Primary Negative Symptoms in Schizophrenia, “A Systematic Review”, Schiozopherma Research 88 (1-3), Page 5-25.

[19] Mustapha, R.O (2001), The Easir Approach to Pharmacology for all Health Professionals; ISBN, Page 167-182.

[20] Nelson J., Juhan - Vague .I. and Thompson S.G (), Involvement of theHaemostaic system in the Insulin Resistance Syndrome. Arterioscler Thrombin (13) 1865-73.

[21] Ochei, J. and Kolhatkar A., (2008), Medical Laboratory Science, Theory and Practices, Tata McGraw-Hill, Page 311-347.

[22] Osinbowale O. Ali .H., Chi Y.W. (2010), Venous Thromboembolism; A Clinical Review. Postgraduate Medical (122) Page 54-65 (Pubmed).

[23] Owen, R. and Charles, A. (2001), History of Blood Coagulation, May foundation for medical Education and Research, Rochester, Minnesota.

[24] Perkins, D. O. (2002), Predictors of Noncompliance in Patients with Sehizophrenia. Journal Clinic Psychiatry 63(12) Page 11211128 (Pubmed).

[25] Sander, J.W Hart Y.M., Trimble M.R. andShorvon S.D. (1991), Vigabatrin and Psychosis. Journal of Neurology, Neurosurgery and Psychiatric54(5) Pages 435-4391.

[26] Wang, P. S. (2010), Rethinking Mental Illness, The British Journal of Psychiatry. 303 (19) Page 1970-1971.

[27] Zornbery, G.L. and Jick, H. (2000), Antipsychotic Drugs used and the Risk of First-time Idiopathic Venous Thromboembolism, A Case Control Study, Lancet, (356), Page 1219-1223 (Pubmed). 